\title{
Successful desensitization and transplantation of kidney transplant recipients with donor-specific antibodies
}

\author{
Muhammad Tassaduq Khan
}

Department of Renal Transplant Unit, Dow University Hospital, Karachi, Pakistan

Background: Kidney transplantation has indisputably changed the dynamics of renal medicine and restored hope among patients coming across fatal end-stage renal disease (ESRD). However, sensitization of human leukocyte antigen (HLA) hampers kidney transplantation. Our transplant center used a modified desensitization protocol.

Methods: Desensitization protocol in our transplant center encompassed following mentioned strategy to achieve mean fluorescence intensity (MFI) values $<1,000$ and negative complement-dependent cytotoxicity (CDC) crossmatch for both $T$ and $B$ lymphocytes before proceeding for kidney transplantation: two sessions of plasmapheresis on days 1 and $2 \rightarrow$ injection rituximab on day 2 after plasmapheresis $\rightarrow$ no plasmapheresis on day $3 \rightarrow$ eight sessions of plasmapheresis after day 3 and intravenous immunoglobulin (IVIG) $100 \mathrm{mg} / \mathrm{kg} /$ dose after each session of plasmapheresis $\rightarrow$ repeat HLA antibody detection test to confirm if donor-specific antibodies (DSAs) are present against HLA with MFI values $<1,000$ and CDC crossmatch is negative for both T and B lymphocytes; if "No" then continue plasmapheresis sessions with IVIG $100 \mathrm{mg} / \mathrm{kg} / \mathrm{dose}$ till MFI values are $<1000$ and $\mathrm{CDC}$ crossmatch is negative for both $\mathrm{T}$ and B lymphocytes or if "Yes" then proceed for transplantation $\rightarrow$ repeat dose of rituximab post-transplantation.

Results: All the six cases had moderate levels of DSAs against HLA except for one case with MFI value of 17,962. With implementation of our modified desensitization protocol, we achieved low levels of DSAs with MFI value $<1,000$. Patients were successfully transplanted with no adverse outcomes, such as kidney allograft rejection, on follow-up.

Conclusions: In our transplant center, we successfully desensitized and transplanted six HLA sensitized kidney transplant candidates with moderate to high DSAs and T and B lymphocyte positive CDC crossmatch. Our desensitization protocol comprised of multiple plasmapheresis sessions with simultaneous low dose IVIG and rituximab. Upon follow-up, we did not witness any significant transplant related event such as allograft dysfunction or rejection.

Corresponding author: Muhammad Tassaduq Khan

E-mail: Muhammad.tassaduq@duhs.edu.pk

(c) The Korean Society for Transplantation

This is an Open Access article distributed under the terms of the Creative Commons Attribution Non-Commercial License (http://creativecommons.org/licenses/by-nc/4.0/) which permits unrestricted non-commercial use, distribution, and reproduction in any medium, provided the original work is properly cited. 